\title{
Safety of Short-Chain Fructooligosaccharides and GRAS Affirmation by the U.S. FDA
}

\author{
Patrick B. SMITH* \\ GTC Nutrition LLC, 600 Corporate Circle, Suite H, Golden, $C 080403$, USA
}

Presented at International Symposium on Fructooligosaccharides, held in Tokyo, July 3, 2001. Received for publication, February 22, 2002

This paper briefly reviews the general process through which GTC Nutrition Company was able to demonstrate through scientific procedures the safety and naturalness of short-chain fructooligosaccharides ( $\left(\mathrm{SCFOS}^{\circledR}\right)$ to the U.S. Food and Drug Administration (FDA). The result of the successful regulatory petition was an FDA "generally recognized as safe (GRAS)" status of fructooligosaccharides as a food ingredient and consequently a broader market recognition and acceptance of our short-chain fructooligosaccharides as a functional food ingredient.

Key words: safety; scFOS; FDA; regulatory

\section{INTRODUCTION}

The U.S. Food and Drug Administration (FDA), is the federal agency primarily responsible for the safety of foods in the United States. To ensure the safety of these products, the FDA's current regulations require a demonstration of the safety of any ingredient added to conventional foods. Thus ingredients added to foods must go through the FDA's review and approval process as either a 1) food additive or be 2) determined to be "generally recognized as safe" (GRAS) by qualified experts for their intended use (1).

The GRAS standard is defined by regulation as "a reasonable certainty in the minds of qualified scientists that the substance is not harmful under its intended conditions of use" (1). The standard also requires that the data used to provide evidence of safety be generally available and that a consensus exist among qualified experts about the safety of the substances for its intended use.

Food ingredients whose use is generally recognized as safe by qualified experts are not required by law to receive FDA approval before marketing. Under current procedures, a manufacturer may determine that use of a food substance is GRAS without formally petitioning to FDA. However, if a manufacturer wants FDA affirmation of its determination, the company must submit a petition and go through a rule-making process.

Initially in 1992, GTC Nutrition Company (GTC), after extensive scientific testing to demonstrate safety

*Corresponding author. Mailing address: GTC Nutrition LLC, 600 Corporate Circle, Suite H, Golden, CO 80403, U.S.A. Phone: +1-303-216-2489. Fax. +1-303-216-2477. E-mail: generalinfo@gtcnutrition.com and a careful review of existing scientific data by its internal and external scientific staff and advisors, independently determined that the use of its scFOS is GRAS. Thus a self-affirmation as GRAS was established.

Many of its partners and customers agreed with that conclusion, most notably Ross Products, a division of Abbott Laboratories, who has been including NutraFlora ${ }^{\circledR}$ scFOS in a variety of their products for over 5 years.

While currently companies are not required to notify the FDA of their GRAS determinations, in 2000, for a variety of scientific and market reasons, GTC made the decision to voluntarily submit a GRAS determination for review by the FDA.

\section{SCFOS GRAS NOTIFICATION PROCESS}

GTC Nutrition Company's notification that the use of scFOS is exempt from the premarket approval requirements of the Federal Food, Drug, and Cosmetic Act because it has independently and objectively determined that such use is GRAS, included detailed information regarding the identity of GTC's scFOS, its historical exposure and intended use and the technical basis for the GRAS determination.

\section{Identity of scFOS}

The common or trade names for the substance that is the subject of the GRAS determination include: fructooligosaccharide, FOS, short-chain fructooligosaccharide, scFOS ${ }^{\circledR}$, Neosugar, NutraFlora ${ }^{\circledR}$, Meioligo $^{\circledR}$, and Actilight ${ }^{\circledR}$.

The structure of the scFOS are sucrose molecules to which one, two or three additional fructose molecules have been linked in sequence. 
The scFOS is produced using proprietary methods on a commercial scale from sucrose using a fructosyltransferase enzyme produced by Aspergillus japonicus. Made in this way, the scFOS are all natural and identical to scFOS found in foods. It is important to note that the subject of the GRAS notification identified only GTC's short-chain fructooligosaccharide with a degree of polymerization ranging from 3-5. This particular type can only be produced through Meiji Seika Kaisha's enzymatic proprietary manufacturing process. No other oligosaccharides were included in this GRAS determination.

\section{Historical Exposure and Intended Use of scFOS}

First, GTC needed to demonstrate evidence of a substantial history of consumption (naturalness) of scFOS by significant number of consumers.

GTC demonstrated that fructooligosaccharides exist naturally in a variety plants and is consumed by humans as a component of the commonly consumed foods such as onions, bananas, lettuce and wheat. GTC estimated that background exposures to scFOS from naturally occurring foods ranges from 145 to $250 \mathrm{mg}$ per person per day at the 90th percentile consumption level (2).

Secondly GTC needed to show the conditions of use for scFOS including the foods in which it is to be used, levels of use in those foods, the purposes for which the substance is used, including, if appropriate, a description of the population expected to consume the substance.

ScFOS is proposed for use as an ingredient in 18 food categories such as nutritional bars, baby foods, biscuits, confectionary and milk beverages (2).

GTC estimated that dietary exposure to fructooligosaccharides from its intended use as a bulking agent and prebiotic which would range from approximately 3.1 to 12.8 grams per person per day at the 90 th percentile consumption level (2).

In addition, scFOS have a long history of use in Japan and Europe, where they are approved for use in over 500 processed food products ranging from infant formulas to confectionery products.

\section{Technical Evidence of Safety of scFOS}

The basis for the GRAS determination for the scFOS was based on a combination of scientific procedures and common use in food.

An extensive database, consisting of both animal and human exposure and safety data, is available for determination of the safety of scFOS for its proposed uses as an ingredient in foods at the levels specified in the GRAS notification.

Studies on the metabolic fate of scFOS indicate that it is virtually unabsorbed and undigested by enzymes. A very small amount is hydrolyzed by stomach acid and absorbed into the body as fructose and glucose. The majority of undigested scFOS passes unchanged into the colon where it is fermented by the microflora into gases and short-chain fatty acids (SCFA) (2).

The physiologic effects of scFOS are similar to those of a fiber and include resistance to digestion by enzymes, fermentation by colonic microflora, shortened gastrointestinal transit time, increased fecal weight, reduction of fecal $\mathrm{pH}$, predictable reduction in caloric value, reduction of plasma cholesterol and triglycerides, and reduction in glucose absorption (2).

The safety of scFOS was corroborated by examining its impact on mineral absorption, nitrogen balance and colonic epithelial cells. ScFOS does not have a detrimental effect on mineral absorption. Indeed, B 2-1 fructans have been shown to have a positive impact on calcium and magnesium absorption and balance. The effect of scFOS on nitrogen utilization and excretion indicates that there are no safety concerns, and that there is potential benefit due to the increase in nitrogen excretion in the feces, enhanced urea nitrogen transfer into the large intestine, and enhanced bacterial utilization of ammonia nitrogen. ScFOS has been shown to produce a trophic effect on colonocytes, and studies in rats have demonstrated that scFOS can produce colon tumor inhibition (2).

The systemic effect produced by scFOS include its modulation of both blood glucose and lipid levels. ScFOS ingestion does not increase plasma glucose or stimulate secretion of insulin. In addition, similar to other soluble dietary fibers, scFOS has been shown to have both hypolipidemic and hypocholesterolemic effects. Therefore, there is no concern that scFOS ingestion may adversely affect either glycemic control or blood lipid levels (2).

Toxicological studies indicate that scFOS is not mutagenic or teratogenic and does not produce significant adverse effects or carcinogenicity in animals following chronic administration at levels up to 15 percent $(2,664 \mathrm{mg} / \mathrm{kg} / \mathrm{day})$ in the diet. The effects that were noted in animal studies are consistent with gastrointestinal disturbances caused by high levels of any nondigestible material (2).

Human tolerance to consumption of scFOS has been established in a number of clinical trials. Study results indicate that scFOS, ingested at up to $20 \mathrm{~g} /$ day in adults, 
appears to be safe and well tolerated. In addition, a study reporting the health effects of scFOS consumption by infants in 90th percentile consumptions of 3.0 and 4.2 g scFOS/day, respectively. Therefore, safety (and tolerance) of the B 2-1 fructans has been established, not only in adults, but in infants and children as well. Based on human clinical data, the Acceptable Intake Level (AIL) for scFOS ingestion for the general population, excluding infants less that one year of age, is determined to be $20 \mathrm{~g} /$ day; the AIL for infants less than one year old is determined to be $4.2 \mathrm{~g} /$ day (2).

After reviewing the notification, the FDA determined it provided a sufficient basis for a GRAS determination. The FDA's response to the notification indicated that, in light of the data and information presented, it had no questions, in which case GTC could continue to market the product without concern over FDA enforcement actions.

\section{MARKET IMPLICATIONS OF SCFOS GRAS RECOGNITION}

GTC made the decision to voluntarily submit its GRAS determination because the market and regulatory conditions in the United States for the industry's GTC serves were beginning to change. Demand for functional foods, with a market in the United States of America at US\$17.2 billion (3), or foods that provide the basic attributes of traditional foods (taste, aroma, or nutritive value) but also claim to provide an additional health benefit, were and are increasing dramatically. With the increased demand has come increased scrutiny from the scientific and regulatory community about whether these foods and beverages are actually safe to use. In addition, a number of reputable companies in the food and feed industry, while quite eager to participate in this burgeoning market, are hesitant to engage in commercial development of products containing ingredients that are not recognized as safe by the FDA. Thus to allay any concerns potential customers or regulatory authorities might have regarding GTC's ingredient and to scientifically differentiate GTC's scFOS in the marketplace, GTC decided to utilize independent scientific and legal review of GTC's scFOS and its intended use to confirm its GRAS determination.

GTC Nutrition Company is quite proud of its and its partner's past and current work regarding scFOS, specifically Meiji Seika Kaisha and Beghin Meiji Industries.

Since the successful GRAS notification GTC has seen an increased positive awareness of its product in the marketplace and a corresponding increase in revenues with new customers, most notable Nestle North America. GTC is hopeful to continue that trend.

\section{REFERENCES}

(1) FDA Code of Federal Regulations. 1997. Federal Register 62 (74): 18937-18964.

(2) GTC Nutrition Company. 2000. Generally Recognized as Safe Notification for Short-Chain Fructooligosaccharide. p. 1-107.

(3) Nutrition Business Journal. 2001. Annual overview of the nutrition industry. Volume VI. 